\title{
Optimal Commutation Laws in the Frequency Domain For PM Synchronous Direct-Drive Motors
}

\author{
Farhad Aghili, Member, IEEE, Martin Buehler, Member, IEEE, and John M. Hollerbach, Fellow, IEEE
}

\begin{abstract}
This paper presents a new model for torque generation of permanent-magnet synchronous motors and their control based on Fourier coefficients. A new commutation strategy delivers ripple-free torque and simultaneously minimizes copper losses for the case when the motor's servo amplifier dynamics are negligible. However, multipole motors give rise to high frequency control signals, and often the dynamics of the current amplifier are no longer negligible. For this case, we present a new commutation strategy which minimizes the torque ripple and the velocity induced change in the torque transfer function. The performance of both commutation laws is validated via simulations based on experimental data from a synchronous motor. Minimal computational requirements make these algorithms ideal for real time implementation.
\end{abstract}

Index Terms-Motor drives, permanent magnet motors, synchronous motors, torque control.

\section{INTRODUCTION}

A CCURATE and high bandwidth sources of torque are essential for the precise position, velocity or force control necessary in many robotics and automation applications. Direct drive motors, and in particular synchronous motors [1], are ideal candidates for these tasks since they eliminate the nonlinear backlash, friction and cogging effects inherent in mechanical transmissions which can severely compromise performance [2]. In addition, the performance of positive joint torque feedback systems [3] critically relies on the capability to produce actuator torque accurately.

The control problem of translating a desired torque command faithfully into a motor torque and the underlying motor model have been studied by several researchers [4]-[11]. Manzer et al. [4] characterized variable-reluctance motors by approximating their flux linkage through piecewise fits of polynomials. Murai et al. [12] proposed heuristic commutation for nonsinusoidal flux distribution. Starr and Wilson [13] and Newman and Patel [14] applied a 2-D lookup table and a multivariable function to determine the phase currents of a variable-reluctance motor with respect to position and torque set points. Filicori et al. [6] proposed a dynamical torque controller based on a flux observer minimizing copper losses or the maximum motor-feeding

\footnotetext{
Manuscript received February 21, 2000; revised July 11, 2000. This work was supported in part by the PRECARN TDS Project, MPB Technologies of Montreal, PQ, Canada. Recommended by Associate Editor W. M. Portnoy.

F. Aghili is with the Canadian Space Agency, Saint-Hubert, PQ J3Y 8Y8, Canada.

M. Buehler is with the Department of Mechanical Engineering, McGill University, Montréal, PQ H3A 2A7, Canada (e-mail: buehler@ cim.mcgill.ca).

J. M. Hollerbach is with the Department of Computer Science, University of Utah, Salt Lake City, UT 84112 USA.

Publisher Item Identifier S 0885-8993(00)09806-9.
}

voltage. Le-Huy et al. [15] and Favre et al. [16] compromise the torque ripple harmonics for brushless DC motor by using several drive current waveforms. A similar approach to ours, reducing torque ripple and minimizing copper losses based on Fourier coefficients in brushless permanent magnet motors has been proposed by Hung and Ding [17]. However the approach does not consider the velocity induced torque ripple, caused by limited current amplifier bandwidth. Jahns and Soong [10] suggested that pulsating torque can be eliminated by improving machine design such as skewing and fractional slot pitch windings. It also reviews control-based techniques for compensation of torque-ripple through a torque estimator. A fast ripple compensation scheme is proposed by Holtz and Springob [11] that is based on a model of parasitic effects, for which a complex state-variable approach is used. Moreover, a high bandwidth ripple compensation is enabled by using a deadbeat current controller and current predictor. The main trend in the control of synchronous motors is based on transforming currents via the ' $d-q$ transformation' [18]. Since the transformation linearizes only an ideal motor with perfectly sinusoidally distributed magneto-motive force, another torque set point is cascaded to cancel torque ripples [7], [19]. This method has been successfully implemented on the previous version of the McGill/MIT motor at MIT [19]. Less than $\pm 1 \mathrm{Nm}$ torque ripple has been reported at low speed, however no attempts to cancel torque ripple at high speed or to minimize power dissipation were made.

This paper presents a new open-loop torque control approach for permanent magnet synchronous motors. The problem is how to modulate the phase currents as a function of the angular motor position such that the desired instantaneous torque is generated, with minimum power dissipation. To minimize the deteriorating effect of motor velocity on the generation of torque ripple, the dynamics of the power amplifier is also taken into account in the commutation design.

A new approach to torque modeling and control where both motor torque function and commutation strategy are expressed by complex truncated Fourier series is presented in Section II. Since both torque and commutation functions are periodic nonlinear functions, sinusoidal bases offer a very concise representation. In contrast, describing a function by a look-up table may require a vast amount of data. Moreover, the phase torques are shifted versions of each other. This feature can be exploited nicely in the frequency domain to simplify the analysis and design of the commutation. In addition, the Fourier coefficients of the torque function can be extracted on-line based on phase voltage measurements only [20].

The most important aspect of this approach however, and the subject of Section III, is that it is possible to relate the Fourier 


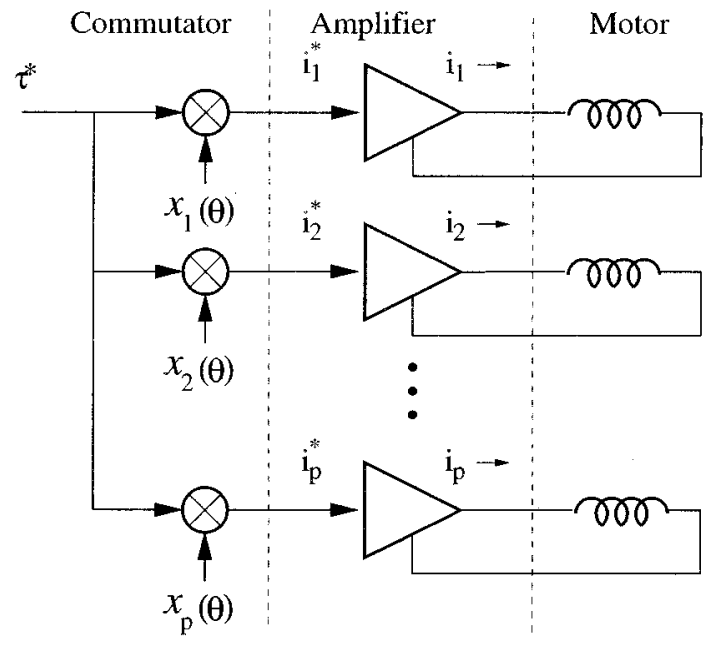

Fig. 1. Motor-amplifier-control system.

series coefficients of the phase torque function and those of the commutation function by a set of linear equations. When the amplifier bandwidth is sufficiently high, the solution yields ripple free torque and minimizes copper losses. However, when the amplifier bandwidth is limited, ripple free torque can be accomplished only at one particular velocity. Consequently we propose another commutation law which minimizes the root-meansquare (rms) norm of the torque ripple over a range of selected velocities. It is worthwhile pointing out that the delays attributed to the digital controller implementation can be lumped with the transfer function of the current amplifier. Section IV demonstrates the effectiveness of the two commutation laws by comparing their torque ripple as a function of motor velocity to that of the standard sinusoidal commutation.

\section{TORQue Modelling IN THE FREQUENCY DOMAIN WITH INCLUSION OF AMPLIFIER DYNAMICS}

The structure of the motor torque controller for a general synchronous motor is shown in Fig. 1. We should note that the McGill/MIT motor [1] is the experimental system which motivated this research. It is a wound-field synchronous motor with identical rotor and stator armatures. Due to practical limitations of the motor prototype, the phase currents of the rotor armature were set constant independent of the torque set point. That is, the motor exactly resembles a permanent magnet synchronous motor on which the analysis and control design are based herein, yet the developed controller is extendible to wound-field synchronous motors. The torque generated by a single phase is a function of the phase current, $i_{r}$, and the motor angle, $\theta$. For a complete list of variables, see Table I. In the linear magnetic regime the function is linear in current, and the total torque $\tau$ is the sum of the phase torque contributions

$$
\tau=\sum_{r=1}^{p} \tau_{r}=\sum_{r=1}^{p} i_{r} y_{r}(\theta) .
$$

Since successive phase windings are shifted by $2 \pi / p$, we have the relationship,

$$
y_{r}(\theta)=y(q \theta+2 \pi(r-1) / p),
$$

where $y(\theta)$ is the motor's torque function and $q$ is the number of motor poles. The commutator commands the phase currents, $i_{r}^{\star}$, via

$$
i_{r}^{\star}\left(\tau^{\star}, \theta\right)=\tau^{\star} x_{r}(\theta) .
$$

The individual phase control signals can be expressed based on the periodic commutation function $x(\theta)$,

$$
x_{r}(\theta)=x(q \theta+2 \pi(r-1) / p),
$$

where $N$ is chosen such that $2 N / p$ is an integer. Since both $x(\theta)$ and $y(\theta)$ are periodic functions with position periodicity of $2 \pi / q$, they can be approximated effectively via the truncated complex Fourier series

$$
x(\theta)=\sum_{n=-N}^{N} c_{n} e^{j n q \theta}, \quad y(\theta)=\sum_{m=-N}^{N} d_{m} e^{j m q \theta}
$$

where $N$ can be chosen arbitrary large, but $2 N / p$ must be an integer. Since both are real valued functions, their negative Fourier coefficients are the conjugate of their real ones, $c_{-n}=\bar{c}_{n}$ and $d_{-n}=\bar{d}_{n}$. Since the magnetic force is a conservative field for linear magnetic systems, we have $\oint \tau_{j}(\theta) \mathrm{d} \theta=0$ which implies zero average torque over a period, and thus $c_{0}=0$.

The motor model and its control can be described by the vectors $\mathbf{c}, \mathbf{d} \in \mathbb{C}^{N}$ of the Fourier coefficients of $x(\theta)$ and $y(\theta)$, respectively, by

$$
\begin{aligned}
\mathbf{c} & =\left[c_{1}, c_{2}, \cdots, c_{N}\right]^{T}, \\
\mathbf{d} & =\left[d_{1}, d_{2}, \cdots, d_{N}\right]^{T} .
\end{aligned}
$$

The control problem is to find the vector of the commutation spectrum, $\mathbf{c}$, with respect to the torque spectrum vector $\mathbf{d}$, such that the motor torque $\tau$ becomes ripple free, i.e., independent of the motor angle $\theta$. With $h(t)$ defined as the impulse response of the current amplifiers, we have

$$
i_{r}(t)=\int_{0}^{t} i_{r}^{\star}(\zeta) h(t-\zeta) \mathrm{d} \zeta .
$$

After substituting (3) and (6) into (1), the total motor torque can be expressed as

$$
\begin{array}{r}
\tau\left(\tau^{\star}, \theta\right) \\
=\sum_{r=1}^{p}\left(\left(\sum_{m=-N}^{N} d_{m} e^{j m(q \theta(t)+2 \pi(r-1 / p))}\right) \int_{0}^{t} \tau^{\star}(\zeta)\right. \\
\left.\cdot \sum_{n=-N}^{N} c_{n} e^{j n(q \theta(\zeta)+2 \pi(r-1 / p))} h(t-\zeta) \mathrm{d} \zeta\right) \\
=\int_{0}^{t} \tau^{\star}(\zeta)\left(\left(\sum_{n=-N}^{N} \sum_{m=-N}^{N} c_{n} d_{m} e^{j q(n \theta(\zeta)+m \theta(t))}\right)\right. \\
\left.\cdot \sum_{r=1}^{p} e^{2 \pi j((r-1) / p)(n+m)}\right) h(t-\zeta) \mathrm{d} \zeta
\end{array}
$$

This expression can be simplified by noting that the third summation term in the right-hand side of (7) vanishes when $l=$ $m+n$ is not a multiple of the number of phases $p$

$$
\sum_{r=1}^{p} e^{2 \pi j l((r-1) / p)}= \begin{cases}p, & \text { if } l=0, \pm p, \pm 2 p, \pm 3 p, \cdots \\ 0, & \text { otherwise }\end{cases}
$$


TABLE I

NOMENCLATURE

\begin{tabular}{|c|c|}
\hline c & vector of Fourier coefficients of the commutation function \\
\hline d & vector of Fourier coefficients of the phase torque function \\
\hline$g(t), G(s)$ & motor torque response in the time domain and the Laplace domain \\
\hline$h(t), H(s)$ & current servo response in time domain and Laplace domain \\
\hline$i_{r}, i_{r}^{\star}$ & $r$ th phase current and commanded $r$ th phase current \\
\hline$j$ & $\sqrt{-1}$ \\
\hline $\mathbf{k}$ & vector contains the Fourier coefficient of torque ripple \\
\hline$p$ and $q$ & number of phases and poles \\
\hline$P_{\text {loss }}$ & motor power losses \\
\hline$R$ & armature resistance \\
\hline$x_{r}(\theta)$ & rth phase commutation function \\
\hline$y_{r}(\theta)$ & $r$ th phase torque function \\
\hline$\omega$ & frequency \\
\hline$\theta$ & motor angle \\
\hline$\tau$ & motor torque \\
\hline$\tau_{l i n}$ & position-independent part of motor torque \\
\hline$\tau_{\text {rip }}$ & motor torque ripple \\
\hline$\tau^{\star}$ & command torque \\
\hline$\nu$ & motor velocity \\
\hline $\operatorname{Re}, \operatorname{Im}$ & the real and imaginary parts of a complex number \\
\hline $\bar{c}$ & complex conjugate \\
\hline $\mathbf{a}^{T}$ & transpose of a vector \\
\hline$\langle\mathbf{a}, \mathbf{b}>$ & inner product in complex field: $\mathbf{a} \cdot \overline{\mathbf{b}}$ \\
\hline$|\cdot|$ & absolute value of a complex number \\
\hline$\|\mathbf{a}\|$ & Euclidian norm of a vector: $\sqrt{\mathbf{a}^{T} \mathbf{a}}$ \\
\hline$\|u\|_{r m s}$ & root-mean-square norm of a signal: $\frac{1}{T}\left(\int_{0}^{T} u^{2}(t) d t\right)^{1 / 2}$ \\
\hline
\end{tabular}

At constant velocities, $\nu=d \theta / d t$, we have $\theta(t)-\theta(\zeta)=$ $\nu \cdot(t-\zeta)$. Defining $\Omega=q \nu$ and $F=p q$, and swapping integration and summation, the torque (7) can written by

$$
\begin{aligned}
& \tau\left(\tau^{\star}, \theta\right)=p \sum_{\substack{n=-N \\
n \neq 0}}^{N} \sum_{l=(n-N) / p}^{(n+N) / p} c_{n} d_{p l-n} e^{j F l \theta} \\
& \cdot\left(\int_{0}^{t} \tau^{\star}(\zeta) e^{-j \Omega n(t-\zeta)} h(t-\zeta) \mathrm{d} \zeta\right)
\end{aligned}
$$

The torque ripple, $\tau_{\text {rip }}\left(\tau^{\star}, \theta\right)$, is defined as

$$
\tau_{r i p}\left(\tau^{\star}, \theta\right)=\tau\left(\tau^{\star}, \theta\right)-\tau^{\star}
$$

while the position independent part of the produced torque in (8) is defined as

$$
\tau_{l i n}\left(\tau^{\star}\right)=p \sum_{\substack{n=-N \\ n \neq 0}}^{N} \bar{c}_{n} d_{n} \int_{0}^{t} \tau^{\star}(\zeta) e^{-j \Omega n(t-\zeta)} h(t-\zeta) \mathrm{d} \zeta .
$$

\section{Design of Commutation LaWs in the FREQUENCY-DOMAIN}

\section{A. High Amplifier Bandwidth: First Commutation Law}

In the absence of significant current amplifier dynamics, the commutation problem has infinitely many solutions. In this case, it is possible to minimize the power dissipation. 
Proposition: Let vector $\mathbf{c}$ represent the Fourier coefficients of the commutation function. Then the power loss is proportional to $\|\mathbf{c}\|^{2}$, where $\|\cdot\|$ depicts the Euclidean norm of a vector.

Proof: Suppose $R$ is the armature resistance. Then the average of dissipated power over time period $T$ is

$$
P_{\text {loss }}=\frac{1}{T} \sum_{r=1}^{p} \int_{0}^{T} R i_{r}^{2}(t) d t .
$$

By changing the integral variable from time $t$ to $\theta$, where $d \theta=$ $\nu d t$ and $\nu T=2 \pi / q$, we have

$$
P_{\text {loss }}=\frac{R q}{2 \pi} \sum_{r=1}^{P} \int_{0}^{2 \pi / q} \tau^{\star} x_{r}^{2}(\theta) \mathrm{d} \theta .
$$

By virtue of Parseval's theorem [21], the power loss per unit commanded torque, i.e., $\tau^{\star}=1$, is

$$
P_{\text {loss }}=p R\|\mathbf{c}\|^{2} \text {. }
$$

The immediate consequence of the above formula is that minimizing power loss is tantamount to minimizing the Euclidean norm of the commutation spectrum vector.

We can ignore the dynamics of the amplifier if its bandwidth is much higher than the frequency of the highest harmonics at maximum velocity, that is $h(t)$ decays faster than $e^{-j \Omega N t}$. Now $h(t)=\delta(t)$ and (9) and(10) simplify to

$$
\begin{aligned}
\tau_{\text {rip }} & =\left(\sum_{l=-2 N / p}^{2 N / p} k_{l} e^{j F l \theta}\right) \tau^{\star} \\
\tau_{l i n} & =p k_{0} \tau^{\star} .
\end{aligned}
$$

The terms $k_{l}$ are the Fourier coefficients of the torque ripples which can be calculated as follows:

$$
k_{l}= \begin{cases}p \sum_{n=1}^{N} c_{n} d_{p l-n}+p \sum_{n=1}^{N-p l} \bar{c}_{n} d_{n+p l} & \text { if } l<\frac{N}{p} \\ p \sum_{n=p l-N}^{p l-1} c_{n} d_{p l-n} & \text { otherwise. }\end{cases}
$$

The term $k_{0}$ in (13) is the constant part of the circular convolution of $x(\theta)$ and $y(\theta)$. This, in turn, is equal to twice the real part of the inner product of the vectors $\mathbf{c}$ and $\mathbf{d}$

$$
k_{0}=2 p \operatorname{Re}\langle\mathbf{c}, \mathbf{d}\rangle \text {. }
$$

To characterize the spectrum of the torque ripple, we define vector $\mathrm{k}=\left[k_{0}, k_{1}, k_{3}, \cdots, k_{2 N / p}\right]^{T} \in \mathbb{C}^{1+(2 N / p)}$ which contains the Fourier coefficients of torque ripple.

Remark: Analogous to proposition 1, one can show that the root-mean-square norm of the torque ripple is proportional to $\|\mathbf{k}\|$.

$$
\left\|\tau_{\text {rip }}\right\|_{\mathrm{rms}}^{2}=\frac{1}{T} \int_{0}^{T} \tau_{\text {rip }}(t)^{2} d t=\|\mathbf{k}\|^{2} .
$$

In order to eliminate the ripple torque (12) completely, all coefficients $k_{l}$ except $k_{0}$ must be zero. Therefore, from (15) and (14) the optimal commutation strategy, c, must solve

$$
\begin{gathered}
\min \|\mathbf{c}\| . \\
\mathbf{A c}+\mathbf{B} \overline{\mathbf{c}}=\mathbf{k}
\end{gathered} .
$$

The matrices $\mathbf{A}, \mathbf{B} \in \mathbb{C}^{(2(N / P)+1) \times N}$ can be constructed from the torque spectrum vector $\mathbf{d}$ in (5) and (14). For example, for a three phase motor $(p=3)$, see (18) and (19) shown at the bottom of the next page. By separating the real and imaginary parts, (17) can be rewritten in the standard linear form as

$$
\left[\begin{array}{ll}
\operatorname{Re}[\mathbf{A}+\mathbf{B}] & \operatorname{Im}[\mathbf{B}-\mathbf{A}] \\
\operatorname{Im}[\mathbf{A}+\mathbf{B}] & \operatorname{Re}[\mathbf{A}-\mathbf{B}]
\end{array}\right]\left[\begin{array}{l}
\operatorname{Re} \mathbf{c} \\
\operatorname{Im} \mathbf{c}
\end{array}\right]=\left[\begin{array}{l}
\operatorname{Re} \mathbf{k} \\
\operatorname{Im} \mathbf{k}
\end{array}\right] .
$$

This equation can be expressed concisely as

$$
\mathbf{Q}_{1} \hat{\mathbf{c}}=\hat{\mathbf{k}}
$$

where

$$
\hat{\mathbf{c}}=\left[\begin{array}{l}
\operatorname{Re} \mathbf{c} \\
\operatorname{Im} \mathbf{c}
\end{array}\right] \text { and } \hat{\mathbf{k}}=\left[\begin{array}{l}
\operatorname{Re} \mathbf{k} \\
\operatorname{Im} \mathbf{k}
\end{array}\right]
$$

and where $\hat{\mathbf{c}} \in \mathbb{R}^{2 N}$ is the set of unknown control coefficients. The elements of matrix $\mathbf{Q}_{1} \in \mathbb{R}^{(4(N / p)+2) \times 2 N}$ can be obtained based upon the given torque functions, Fourier coefficients according to (20), (18) and (19). Now (21) in conjunction with Proposition 1 can be used to calculate the spectrum of the first commutation. A ripple-free commutation implies that all coefficients $k_{l}$ except $k_{0}$ are zero and $k_{0}=1$, where $\mathbf{e}_{1}=[1,0,0, \cdots, 0]^{T}$. Hence, (21) yields

$$
\mathbf{Q}_{1} \hat{\mathbf{c}}=\mathbf{e}_{1}
$$

where $\mathbf{Q}_{1} \in \mathbb{R}^{(4(N / p)+2) \times 2 N}, \mathbf{e}_{1} \in \mathbb{R}^{(4 N / p)+2}$. In general, for motors with more than two phases, $p>2$, there are fewer equations than unknowns in (23). Therefore, if $\mathbf{e}_{1}$ belongs to the range of $\mathbf{Q}_{1}$, a unique solution is not expected. Instead, a family of solutions which belongs to the null space of $\mathbf{Q}_{1}$ plus a particular solution exist. Among all the solutions, the shortest one in the sense of Euclidean norm is desirable. This is consistent with minimizing power losses, since

$$
\|\hat{\mathbf{c}}\|=\|\mathbf{c}\| \text {. }
$$

The generalized inverse offers the solution $\hat{\mathbf{c}}_{1}=$ $\left(\mathbf{Q}_{1}^{T}\left(\mathbf{Q}_{1} \mathbf{Q}_{1}^{T}\right)^{-1}\right) \mathbf{e}_{1}$ to the problem. However, it may have numerical pitfalls in practice because $\mathbf{Q}_{1} \mathbf{Q}_{1}^{T}$ is usually ill-conditioned. Singular Value Decomposition is a standard technique to deal with such problems [22]. The matrix $\mathbf{Q}_{1}$ can be written as $\mathbf{U} \Sigma \mathbf{V}^{T}$ where $\mathbf{U}$ and $\mathbf{V}$ are orthogonal matrices and $\mathbf{\Sigma}$ is a diagonal matrix whose elements are the singular values of matrix $\mathbf{Q}_{1}$. Since the matrix $\mathbf{Q}_{1}$ is not square, it has to be augmented by adding rows of zeros underneath. The optimal solution, $\hat{\mathbf{c}}_{1}$, is then

$$
\hat{\mathbf{c}}_{1}=\left(\mathbf{V} \boldsymbol{\Sigma}^{-1} \mathbf{U}^{T}\right) \hat{\mathbf{e}}_{1},
$$

where $\Sigma^{-1}=\operatorname{diag}\left\{1 / \sigma_{i}\right\}$ and the inverse of those singular values which are close to zero, is replaced by zero, and $\hat{\mathbf{e}}_{1}$ is an augmented version of $\mathbf{e}_{1}$. The columns of $\mathbf{V}$ corresponding to the zeroed $\sigma_{i}$ construct the bases for the null space of $\mathbf{Q}_{1}$ which shows the class of ripple-free commutation law spectrum whereas (24) is the optimum one.

\section{B. Limited Amplifier Bandwidth: Second Commutation Law}

1) Torque Transfer Function: The more poles exist in a motor, the higher is its maximum torque. However, using more poles results in a higher drive frequency for a given motor speed. This tends to become difficult for the current servo unit to track the reference current input. Before designing the commutation law, it is necessary to derive the system transfer function in the presence of amplifier dynamics. The commutation law aims at eliminating the torque ripple (9), 
while (10) shows how the torque set point $\tau^{\star}$ is transformed linearly into motor torque. This section is devoted to deriving the torque transfer function of the linear system in the presence of amplifier dynamics. Let $g(t)$ be the impulse function of the system,

$$
\tau_{l i n}(t)=g(t) * \tau^{\star}(t)
$$

Then by virtue of the convolution integral and (10),

$$
g(t)=2 \sum_{n=1}^{N}\left|a_{n}\right| \cos \left(\Omega n t+\angle a_{n}\right) h(t),
$$

where $a_{n}=p c_{n} \bar{d}_{n}$. Thus the impulse response of the torque, $g(t)$, is formed by modulating $\cos \left(\Omega n t+\angle a_{n}\right)$, the carrier, by the amplifier's impulse response, $h(t)$. Modulating a signal by cosine with spectrum $H(j \omega)$ causes replicas of $H(j \omega)$ to be placed at plus and minus the carrier frequency $\omega$. The Fourier transform of the system in terms of the amplifier's frequency response $H(j \omega)$ is

$$
G(j \omega)=\sum_{n=1}^{N} a_{n} H(j(\omega+\Omega n))+\bar{a}_{n} H(j(\omega-\Omega n)) .
$$

The above complex transfer function can be approximated by a simpler one which give insight into the dynamics of torque generation. Using the Taylor expansion of the shifted functions around $\omega$, one can write,

$$
G(j \omega)=k_{0} H(j \omega)+\Delta(j \omega)
$$

where $k_{0}$ is previously defined, and $\Delta(j \omega)$ is a perturbation term which enters as in an additive form into the nominal transfer function $k_{0} H(j \omega)$. The perturbation transfer function can be written up to first-order approximation by

$$
\begin{aligned}
\Delta(j \omega) & \approx 2 \Omega H^{\prime}(j \omega) \operatorname{Im} \sum_{n=1}^{N} n a_{n}, \text { where } \\
H^{\prime}(j \omega) & =\left.\frac{\mathrm{d} H(s)}{\mathrm{d} s}\right|_{s=j \omega} .
\end{aligned}
$$

Therefore, the system transfer function depends on the velocity, $\Omega$, such that at zero velocity it is coincident with that of the current amplifiers, $H(j \omega)$. The above expression also relates the uncertainty of the system to $H^{\prime}(j \omega)$. One can show that for first order or damped second order amplifier dynamics, $\left|H^{\prime}(j \omega)\right|$ is maximum at $\omega=0$. The outcome of this part will be used to design the optimal commutator.

2) Ripple Cancellation: Equation (9) characterizes the torque ripple in the presence of amplifier dynamics. The integral terms indicate that the current still enters linearly. Suppose $\gamma_{n}(t)=h_{a}(t) e^{j n \Omega t}$ is the impulse response of a virtual system associated with the $n$th harmonics. Then

$$
\int_{0}^{t} \tau^{\star}(\zeta) e^{j n \Omega(t-\zeta)} h_{a}(t-\zeta) d \zeta=\gamma_{n}(t) * \tau^{\star}(t)
$$

where $*$ denotes the convolution operator. The exponential function shifts the frequency of the Fourier transform of the current amplifier, $H(j \omega)$. Therefore in the frequency domain we have, $\Gamma_{n}(j \omega)=H(j(\omega+\Omega n))$. The steady-state response of the system to a unit step input is

$$
\lim _{\omega \rightarrow 0} \Gamma_{n}(j \omega)=H(j \Omega n) .
$$

Therefore, substituting (27) into (9) yields the steady-state torque ripple. Moreover, since $\gamma_{n}$ is a real valued function, $H(-j \Omega n)=\overline{H(j \Omega n)}$. Now the spectrum of the steady-state ripple can be found analogously to the previous case at each particular velocity $\Omega_{i}=q \nu_{i}$ as

$$
\mathrm{AH}^{i} \mathbf{c}+\mathbf{B} \overline{\mathbf{H}}^{i} \overline{\mathbf{c}}=p \mathbf{k}^{i}
$$

where $\mathrm{k}^{i}$ is the Fourier coefficient of torque ripple at the $i$ th velocity, and $\mathbf{H}^{i} \in \mathbb{C}^{N \times N}$ is a diagonal matrix defined by

$$
\mathbf{H}^{i}=\operatorname{diag}\left\{H\left(j \Omega_{i}\right), H\left(j 2 \Omega_{i}\right), \cdots, H\left(j N \Omega_{i}\right)\right\} .
$$

Equation (28) is similar to (17) by considering $\mathbf{A} \mathbf{H}^{i}$ and $\mathbf{B} \overline{\mathbf{H}}^{i}$ in (28) as $\mathbf{A}$ and $\mathbf{B}$, respectively. Analogously to (23), we have $\mathbf{Q}_{1}^{i} \hat{\mathbf{c}}=\mathbf{e}_{1}$. As a matter of fact, one can show that (23) is the special case where the velocity is set to zero. Therefore, it can be concluded that the first commutation law is always applicable

$$
\begin{aligned}
\mathbf{A} & =\left[\begin{array}{cccccccccc}
\bar{d}_{1} & \bar{d}_{2} & \bar{d}_{3} & \bar{d}_{4} & \bar{d}_{5} & \bar{d}_{6} & \bar{d}_{7} & \cdots & \bar{d}_{N-1} & \bar{d}_{N} \\
d_{2} & d_{1} & 0 & \bar{d}_{1} & \bar{d}_{2} & \bar{d}_{3} & \bar{d}_{4} & \cdots & \bar{d}_{N-4} & \bar{d}_{N-3} \\
d_{5} & d_{4} & d_{3} & d_{2} & d_{1} & 0 & \bar{d}_{1} & \cdots & \bar{d}_{N-7} & \bar{d}_{N-6} \\
\vdots & \vdots & \vdots & \vdots & \vdots & \vdots & \vdots & \vdots & \vdots & \vdots \\
d_{N-1} & d_{N-2} & d_{N-3} & d_{N-4} & d_{N-5} & d_{N-6} & d_{N-7} & \cdots & d_{1} & 0 \\
0 & 0 & d_{N} & d_{N-1} & d_{N-2} & d_{N-3} & d_{N-4} & \cdots & d_{4} & d_{3} \\
\vdots & \vdots & \vdots & \vdots & \vdots & \vdots & \vdots & \vdots & \vdots & \vdots \\
0 & 0 & 0 & 0 & 0 & 0 & 0 & \cdots & 0 & d_{N}
\end{array}\right], \\
\mathbf{B} & =\left[\begin{array}{ccccccc}
d_{1} & d_{2} & d_{3} & d_{4} & d_{5} & \cdots & d_{N} \\
d_{4} & d_{5} & d_{6} & d_{7} & d_{8} & \cdots & 0 \\
d_{7} & d_{8} & d_{9} & d_{10} & d_{11} & \cdots & 0 \\
\vdots & \vdots & \vdots & \vdots & \vdots & \vdots & \vdots \\
d_{N-2} & 0 & 0 & 0 & 0 & \cdots & 0 \\
0 & 0 & 0 & 0 & 0 & \cdots & 0 \\
\vdots & \vdots & \vdots & \vdots & \vdots & \vdots & \vdots \\
0 & 0 & 0 & 0 & 0 & \cdots & 0
\end{array}\right]
\end{aligned}
$$


at every particular velocity. Such a commutation is useful for velocity regulator applications where the velocity remains in the vicinity of an operating point. Since the matrix $\mathbf{Q}_{1}^{i}$ changes with velocity, we can write the equations over an arbitrary number of velocities

$$
\mathbf{Q} \hat{\mathbf{c}}=\mathbf{e}
$$

where $\mathbf{Q}^{T}=\left[\mathbf{Q}_{1}^{1 T}, \mathbf{Q}_{1}^{2 T}, \cdots, \mathbf{Q}_{1}^{s T}\right]$ and $\mathbf{e}^{T}=$ $\left[\mathbf{e}_{1}^{T}, \mathbf{e}_{1}^{T}, \cdots, \mathbf{e}_{1}^{T}\right]$. In this case, there are more equations than unknowns in (29). Therefore, the equations are inconsistent and no solution exists. This implies that it is not possible to eliminate the harmonics at all velocities. Yet, in general, we can find the solution using all equations to minimize the error in the least square sense. Suppose $w_{r}$ and $w_{p}$ are two positive scalars, then we reformulate our problem in (29) as

$$
\left[\begin{array}{c}
\mathbf{Q} \\
\mathbf{I}
\end{array}\right] \hat{\mathbf{c}}=\left[\begin{array}{l}
\mathbf{e} \\
\mathbf{0}
\end{array}\right]
$$

which can be written concisely $\mathbf{Q}_{2} \hat{\mathbf{c}}=\mathbf{e}_{2}$, and $\mathbf{I}$ is an identity matrix. Define error $\epsilon=\mathbf{Q} \hat{\mathbf{c}}-\mathbf{e}_{2}$. The pseudo-inverse [23] offers a solution $\hat{\mathbf{c}}_{2}=\left(\left(\mathbf{Q}_{2}^{T} \mathbf{W} \mathbf{Q}_{2}\right)^{-1} \mathbf{Q}_{2}^{T} \mathbf{W}\right) \mathbf{e}_{2}$ is the weighted least-squares solution minimizing $\|\boldsymbol{\epsilon}\|_{W}^{2}=\boldsymbol{\epsilon}^{T} \mathbf{W} \boldsymbol{\epsilon}$, where $\mathbf{W}$ is a diagonal matrix-again, the singular value decomposition is another technique which can be applied here to cope with ill-conditioning. The minimization criteria is interpreted in the forgoing analysis. Assume that matrix $\mathbf{W}$ is chosen so that scalars $w_{r}$ and $w_{p}$ weigh the error associated with the first and the second blocks of (30). Then

$$
\|\epsilon\|_{W}^{2}=w_{r} \sum_{i=1}^{s} \sum_{j=0}^{2 N / p}\left|k_{j}^{i}\right|^{2}+w_{p} \sum_{j=1}^{N}\left|c_{j}\right|^{2} .
$$

Recall from (28) that $\mathbf{Q}_{1}^{i} \hat{\mathbf{c}}-\mathbf{e}_{1}$ is a vector containing the real and imaginary part of the ripple spectrum at $i$ th velocity. Now, by virtue of proposition 1 and remark 1 ,

$$
\|\boldsymbol{\epsilon}\|_{W}^{2}=w_{r} \sum_{i=1}^{s}\left\|\tau_{\text {rip }}^{i}\right\|_{\mathrm{rms}}^{2}+w_{p} P_{\text {loss }}
$$

where $\tau_{\text {rip }}^{i}$ is the torque ripple at $i$ th velocity. The above expression implies that the second commutation law minimizes the weighted sum of power loss and the rms norm of torque ripple.

\section{IMPLEMENTATION ISSUES AND SIMULATION RESULTS}

The experimental setup is a hydraulic dynamometer testbed consisting of our direct-drive motor coupled to a hydraulic rack and pinion rotary motor (Parker 113A129BME) via a torque transducer (Himmelstein MCRT 2804TC) [24]. An optical encoder (Gurley 8321-4500-CBQA-NB) mounted on the motor shaft measures motor angle. The speed of the hydraulic motor is regulated by a pressure compensated flow control valve (Parker TPCS600S01). The motor torque is measured in a quasistatic condition, where the motor velocity is kept sufficiently low $(1(\operatorname{deg} / s))$, to ensure that inertial torques do not interfere with the measurements. The dynamometer is interfaced to an IBM compatible $66 \mathrm{MHz} 80486$ computer. Despite this limited processing power, the CPU time taken up for the proposed

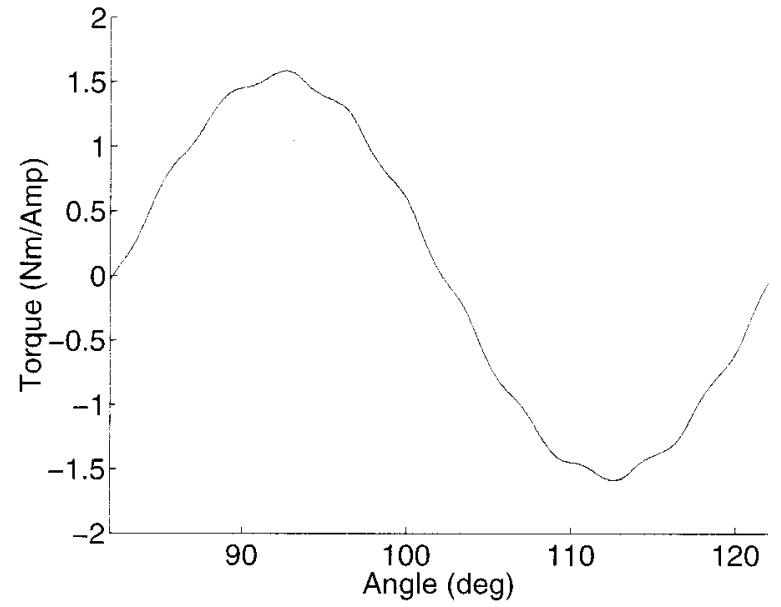

Fig. 2. Torque-angle profile obtained from experimental data.

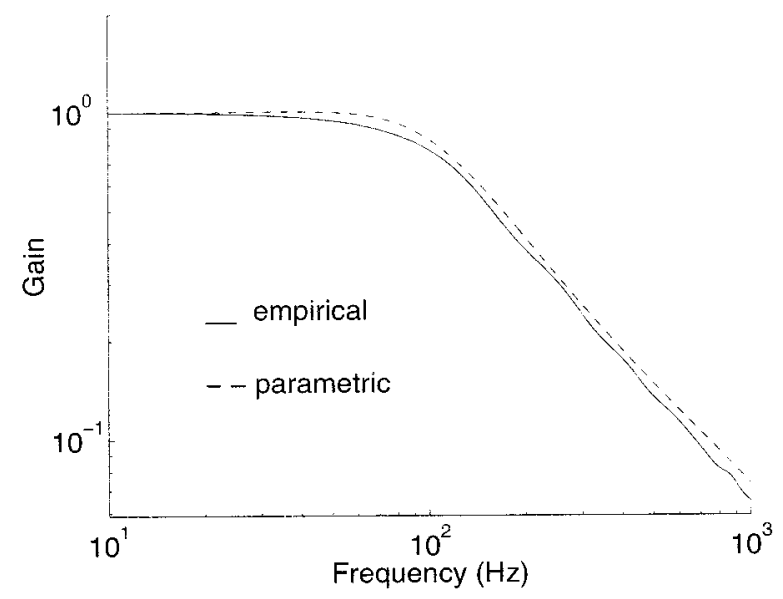

Fig. 3. Frequency response of the current controller.

commutation laws for a three phase motor is only $106 \mathrm{~ms}$, based on Fourier coefficients up to 24 harmonics.

Implementation of the first commutation law only requires the motor's torque-angle profile (phase torque function). We characterized the motor prototype by energizing one phase with a constant current and recording torque measurements versus motor angle using a specially designed dynamometer [24]. The resulting torque-angle profile is shown in Fig. 2. Alternatively, the Fourier coefficients $\mathbf{d}$ can be extracted on-line and without the need for elaborate mechanical test fixtures, based on phase voltage measurements only [20]. These experiments also showed an approximately linear relation among phase currents and phase torques, indicating the motor operates in a linear magnetic regime.

In addition to the torque-angle spectrum, the frequency response of the current servo amplifier $H(s)$ is needed to design the second commutation law. It is obtained experimentally by performing a spectral analysis of the input-output of the system. To this end, a pseudo-random signal generated by the computer is input to the amplifier while the winding current is measured by a hall-effect current sensor (Micro Switch model SLB1AD). The empirical transfer function of the system is illustrated in Fig. 3 by the solid lines. The dashed lines represents the best match fourth order model (in the least-square sense) which is 


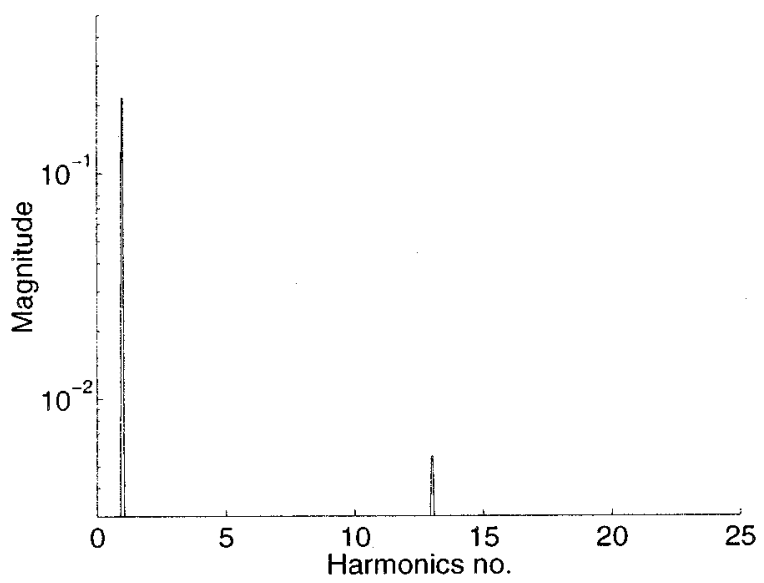

(a)

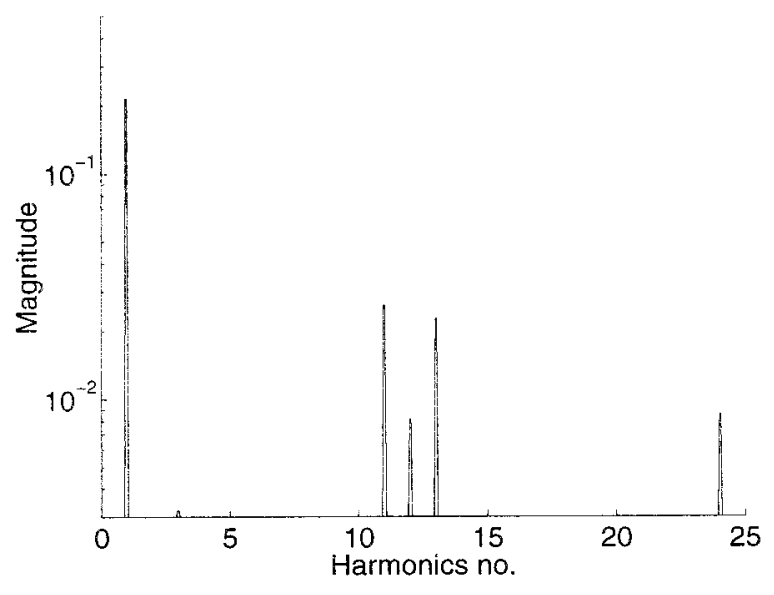

(b)

Fig. 4. Commutation spectra for minimal losses (left) and minimal ripples (right).

used for design and simulation. The loci of the system zeros and poles indicate that the amplifier is a nonminimum phase system with $150 \mathrm{~Hz}$ bandwidth.

Using the theory developed in the previous section and the experimental data, the commutation spectra for minimum power losses and torque ripples were computed and are shown in Fig. 4 which shows the tradeoffs clearly. The first commutator is designed for quasi static operation, while the second one is designed for operating in the velocity range zero to $1(\mathrm{rev} / \mathrm{s})$.

At very low speed, the first commutation law is equivalent to a look-up table based commutation whose performance was reported in [24]. The problem we encountered with our experimental setup was to evaluate the performance of the second commutation law at high velocity. Due to physical constraints, our more motor cannot turn more than one revolution. This prevented us from operating it in steady state in order to eliminate the inertial torque from torque measurement. Therefore, we evaluated the performance of the commutation laws via simulations, based on the experimental motor and current amplifier models.

The value of torque ripples when the first and second commutation laws as well as contemporary sinusoidal commutation are applied is demonstrated in Fig. 5. It is apparent that the first and second commutations do not exhibit torque ripple at

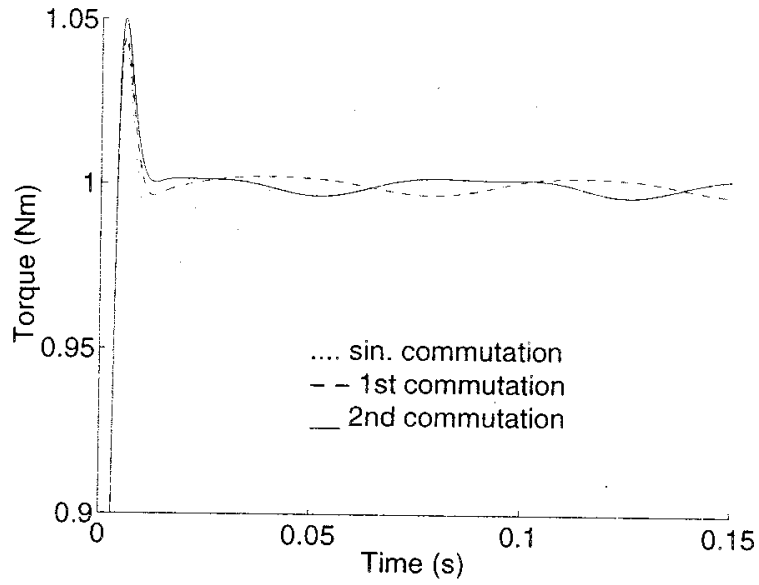

(a)

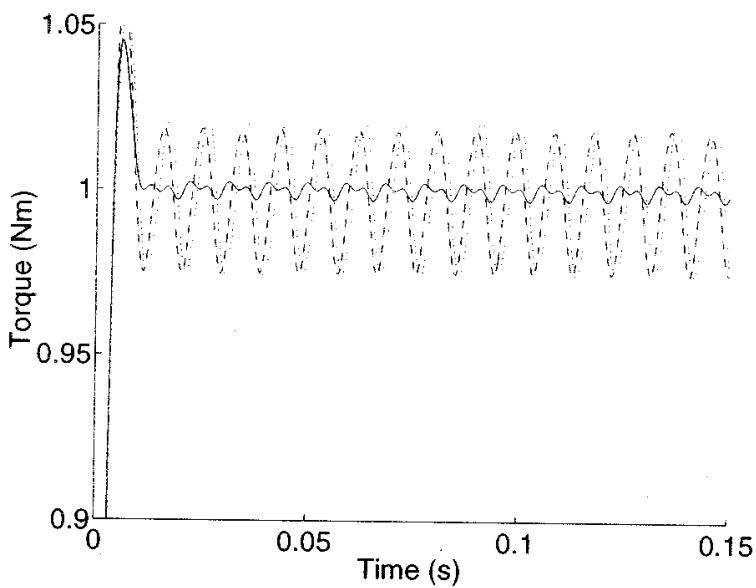

(b)

Fig. 5. Comparison of the induced torque ripples with sinusoidal, the first and second commutations at low $(0.1(r e v / s))$ velocity (left) and high $(1(r e v / s))$ velocity (right).

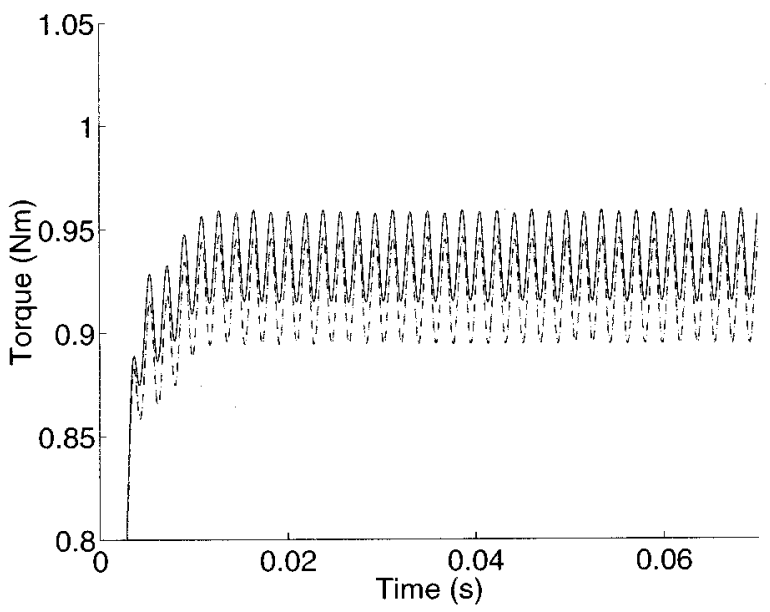

Fig. 6. Torque ripple at $5(\mathrm{rev} / \mathrm{s})$.

low velocity $(0.1(\mathrm{rev} / \mathrm{s}))$ since the maximum current frequency demanded by the commutators is well below the amplifier's cut-off frequency of $150 \mathrm{~Hz}$. However, the spectrum of the first commutation contains the 13th harmonic for torque ripple compensation. Thus at $1(\mathrm{rev} / \mathrm{s})$ the first commutator commands the current amplifier at $117 \mathrm{~Hz}$, which is now close the amplifier 
cut-off frequency. As a result, even though it delivers smooth torque at very low speed, the first commutator causes high ripple at high speed-almost as much as the sinusoidal commutation. The second commutator exhibits very low torque ripple at low and high velocities, although its power loss is slightly higher (4\% more in this particular case). It is important to point out that at even higher velocities, the frequency of the commutation is beyond the bandwidth of the torque controller, and all the commutation exhibits torque ripple. This fact is demonstrated in Fig. 6 that simulates the motor torque at $5(\mathrm{rev} / \mathrm{s})$.

\section{CONCLUSION}

A general model for torque generation of synchronous motors in the presence of current amplifier dynamics is derived. The formulation uses Fourier coefficients to capture the phase torque and commutation function in a concise fashion. In addition, the calculation for commutation is simple and suitable for efficient implementation on DSP processors. We proposed two commutation laws. The first one minimizes copper losses, and can deliver a ripple-free torque independent of motor velocity, provided that the dynamics of the current amplifier are negligible. Otherwise, the motor velocity compromises performance in two ways. First, perfect ripple cancellation is not possible over a range of velocities. Moreover, the torque response of the system varies with velocity. We derived a commutation law addressing both issues. It minimizes the weighted sum of the torque ripple and power losses. Simulations using an experimentally derived torque characterization of a direct drive motor and frequency response current torque characterization of current controller demonstrated the validity of this method.

\section{REFERENCES}

[1] J. M. Hollerbach, I. Hunter, J. Lang, S. Umans, and R. Sepe, "The McGill/MIT direct drive motor project," in Proc. IEEE Int. Conf. Robot. Automat., May 1993, pp. 611-617.

[2] C. H. An, C. G. Atkeson, and J. M. Hollerbach, Model-Based Control of a Robot Manipulator. Cambridge, MA: MIT Press, 1988.

[3] F. Aghili, M. Buehler, and J. M. Hollerbach, "Disturbance attenuation and load decoupling with $\mathrm{h}$ infinity positive joint torque feedback," in Proc. IEEE Int. Conf. Robot. Automat., May 1998, pp. 3613-3618.

[4] D. G. Manzer, M. Varghese, and J. S. Thorp, "Variable reluctance motor characterization," IEEE Trans. Ind. Electron., vol. 36, pp. 56-63, Feb. 1989.

[5] R. S. Wallace and D. G. Taylor, "Low-torque-ripple switched reluctance motors for direct-drive robotics," IEEE Trans. Robot. Automat., vol. 7, pp. 733-742, Dec. 1991.

[6] F. Filicori, C. G. Lo Bianco, and A. Tonielli, "Modeling and control strategies for a variable reluctance direct-drive motor," IEEE Trans. Ind. Electron., vol. 40, pp. 105-115, Feb. 1993.

[7] D. Chen and B. Paden, "Adaptive linearization of hybrid step motors: Stability analysis," IEEE Trans. Automat. Contr., vol. 38, pp. 874-887, June 1993.

[8] D. G. Taylor, "Nonlinear control of electric machines: An overview," IEEE Contr. Syst., vol. 14, pp. 41-51, Nov. 1994.

[9] C. Canudas De Wit and S. I. Seleme, "Robust torque regulation for induction motors: The minimum energy approach," in Proc. 12th World Congr. Int. Fed. Automat. Contr. (IFAC), July 1993, pp. 73-76.

[10] T. M. Jahns and W. L. Soong, "Pulsating torque minimizing techniques for permanent-magnet ac motor drives-a review," IEEE Trans. Ind. Electron., vol. 43, pp. 321-330, Apr. 1996.

[11] J. Holtz and L. Springob, "Identification and compensation of torque ripple in high precision permanent magnet motor drives," IEEE Trans. Ind. Electron., vol. 43, pp. 309-320, Apr. 1996.
[12] Y. Murai, Y. Kawase, K. Ohashi, and K. Okuyama, "Torque ripple improvement for brushless de miniature motors," IEEE Trans. Ind. Applicat., vol. 25, pp. 441-449, June 1993.

[13] G. P. Starr and C. W. Wilson, Design of a Torque Control for Adept-2 Robot. New York: ASME, 1990.

[14] W. S. Newman and J. J. Patel, "Experiments in torque control of the adept-one robot," in Proc. IEEE Int. Conf. Robot. Automat., Apr. 1991, pp. 1867-1872.

[15] H. Le-Huy, R. Perret, and R. Feuillet, "Minimization of torque ripple in brushless dc motor drives," IEEE Trans. Ind. Applicat., vol. IA-22, pp. 748-755, July/Aug. 1986.

[16] E. Favre, L. Cardoletti, and M. Jufer, "Permanent-magnet synchronous motors: A comprehensive approach to cogging torque suppression," IEEE Trans. Ind. Applicat., vol. 29, pp. 1141-1149, Dec. 1993.

[17] Y. Y. Hung and Z. Ding, "Design of currents to reduce toraue ripple in brushless permanent magnet motors," Proc. Inst. Elect. Eng. B, vol. 140, no. 4, 1993.

[18] P. C. Krause, Analysis of Electric Machinery. New York: McGrawHill, 1986.

[19] D. K. Jackson, "Torque-ripple compensation for an axial-air gap synchronous motor,” M.Eng. thesis, Mass. Inst. Technol., Cambridge, Aug. 1994.

[20] F. Aghili, "Design and control of direct-drive systems with applications to robotics," Ph.D. dissertation, McGill Univ., Montreal, PQ, Canada, Oct. 1997.

[21] R. M. Gray and J. W. Goodman, Fourier Transforms. Norwell, MA: Kluwer, 1995.

[22] W. H. Press, B. P. Flannery, S. A. Teukolsky, and W. T. Vetterling, Numerical Recipies in C: The Art of Scientific Computing. Cambridge, U.K.: Cambridge Univ. Press, 1988.

[23] R. C. Dorf, Modern Control Systems. Reading, MA: Addison-Wesley, 1974.

[24] F. Aghili, M. Buehler, and J. M. Hollerbach, "Torque ripple minimization in direct-drive systems," in Proc. IEEE/RSJ Int. Conf. Intell. Syst. Robots, Victoria, BC, Canada, Oct. 1998, pp. 794-799.

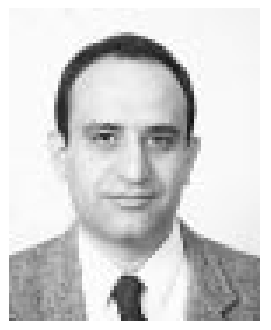

Farhad Aghili (M'91) received the B.Sc. degree in mechanical engineering and the M.Sc. degree in biomedical engineering from the Sharif University of Technology, Tehran, Iran, in 1988 and 1991, respectively, and the Ph.D. degree in mechanical engineering from McGill University, Montreal, PQ, Canada, in 1998.

Currently, he is a Research Scientist with the Canadian Space Agency, where he has been working on the International Space Station program since January 1998. From 1994 to 1997, he was a Research Engineer at MPB Technologies, Montreal. His main areas of interest are mechanical design, mechatronics, control of robotic systems, space robots, and application of robotics in rehabilitation.

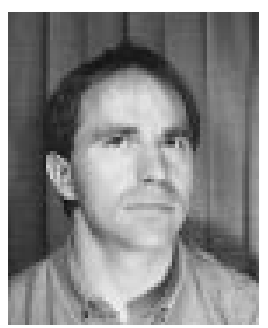

Martin Buehler (M'90) received the Ph.D. degree in electrical engineering from Yale University, New Haven, CT, in 1990, and studied legged locomotion as a Postdoctoral Associate at the Artificial Intelligence Laboratory, Massachusetts Institute of Technology, Cambridge.

In 1991, he joined McGill University, Montreal, PQ, Canada, as a Junior Industrial Research Chair, and is currently an Associate Professor there. His main research interests are in motor control, legged locomotion, biomimetics, energetics, actuators, and robot design and control. He participates regularly in conference organization and reviews for many journals and conferences.

Dr. Buehler is an Associate Editor for the IEEE TRANSACTIONS ON ROBOTICS AND AUTOMATION. 


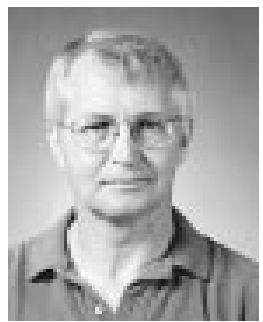

John M. Hollerbach (F’96) received the B.S. degree in chemistry and M.S. degree in mathematics from the University of Michigan, Ann Arbor, in 1968 and 1969, respectively, and the S.M. and Ph.D. degrees in computer science from the Massachusetts Institute of Technology (MIT), Cambridge, in 1975 and 1978, respectively.

He is Professor of Computer Science, and Adjunct Professor of Mechanical Engineering, University of Utah, Salt Lake City. From 1989 to 1994, he was the Natural Sciences and Engineering/Canadian Institute for Advanced Research Professor of Robotics, McGill University, Montreal, PQ, Canada, jointly in the Departments of Mechanical Engineering and Biomedical Engineering. From 1982 to 1989, he was on the faculty of the Department of Brain and Cognitive Sciences and a Member of the Artificial Intelligence Laboratory, MIT; from 1978 to 1982 he was a Research Scientist. Presently, he is Editor of the International Journal of Robotics Research and a Senior Editor of Presence: Teleoperators and Virtual Environments.

Dr. Hollerbach received an NSF Presidential Young Investigator Award, in 1984 and was named a Fellow of the Canadian Institute for Advanced Research, in 1988. He was the Program Chairman of the 1989 IEEE International Conference on Robotics and Automation, a Member of the Administrative Committee of the IEEE Robotics and Automation Society from 1989 to 1993, Technical Editor of the IEEE TRANSACTIONS ON ROBOTICS AND AUTOMATION from 1989 to 1994, and Treasurer of the IEEE/ASME Journal of Microelectromechanical Systems, from 1992 to 1997. He was a Member of the 1994-1995 National Research Council Committee on Virtual Reality Research and Development. He is a Governing Board member of the electronic journal Haptics-e. His research interests combine robotics, virtual reality, and microelectromechanical systems. 